\title{
LA GIMNASIA Y LA ACROBACIA EN EL ÁMBITO EDUCATIVO: ENTREVISTA A LA PROFESSORA DRA ELISA ESTAPÉ TOUS (ESPAÑA)
}

Marco Antonio Coelho Bortoleto, Universidade Estadual de Campinas - UNICAMP, Campinas, São Paulo - Brasil

Teresa Ontañón Barragan, Universidade Estadual de Campinas - UNICAMP, Campinas, São Paulo - Brasil

Durante uma semana do mês de julho nós, do Grupo de Pesquisa em Ginástica da FEFUNICAMP, tivemos a oportunidade de poder trabalhar como uma das mais renomadas treinadoras e pesquisadoras espanholas na área da Ginástica Artística, a Professora Dra. Elisa Estapé Tous da Universidade de León.

Sua experiência como treinadora e como docente no ensino superior e como pesquisadora resultou em significativas contribuições, particularmente na esfera pedagógica, como podemos observar por exemplo nos livros "Las habilidades gimnásticas y acrobáticas en el ámbito educativo" e "La Acrobacia en Gimnasia Artística. Su técnica y su didáctica” publicados em 1999 e 2002 respectivamente.

Durante este encontro, elaboramos uma entrevista que revela aspectos de sua trajetória acadêmica bem como alguns de seus projetos atuais e experiências que acreditamos poder inspirar e contribuir na formação e na atuação dos estudantes e pesquisadores brasileiros desta área. Cabe ressaltar que, decidimos manter a entrevista no idioma original (espanhol), visando garantir a originalidade do depoimento oferecido, o qual agradecemos enormemente de antemão.

\section{¿Podría usted contarnos un poco acerca de su trayectoria académica en la}

\section{Educación Física?}

Empecé como entrenadora de Gimnasia Artística, siendo muy joven en Barcelona, la ciudad donde nací. Tuve algunos buenos resultados con gimnastas hasta que en 1988 lo abandoné y acabé mis estudios de Educación Física que había empezado mucho antes. En 1990, entré en el Instituto Nacional de Educación Física de Castilla y León que 
dependía en aquel entonces de la Junta de Castilla y León y no como actualmente, en la Facultad de Ciencias de la Actividad Física y el Deporte de la Universidad de León. Llegar a ser profesora universitaria había sido uno de mis objetivos desde que era entrenadora y alumna. Y entonces, a nivel de gimnasia, me planteé introducir a los alumnos a la gimnasia con enfoques muy distintos a lo que se había hecho anteriormente y alejados de lo que se conoce en el aprendizaje de la gimnasia en el ámbito competitivo.

Durante más de 10 años usted ha dedicó gran parte de sus esfuerzos académicos a estudiar y enseñar distintas disciplinas gimnásticas y acrobáticas en la Universidad de León. ¿Podría contarnos un poco acerca de estas experiencias docentes, investigaciones y proyectos de extensión?

Sí, me dediqué principalmente a la difusión y divulgación de las habilidades gimnásticas y acrobáticas en el ámbito educativo. En León, hay poca tradición gimnástica y por tanto, pocos alumnos que en primer curso conozcan la gimnasia o hayan aprendido estas habilidades en la Enseñanza Primaria y Secundaria. Por tanto, se intenta atraer a alumnos de otros deportes hacia la gimnasia; esto es tarea difícil pues como sabemos, la gimnasia y la acrobacia son actividades sumamente complejas. Mis objetivos se han centrado desde el primer momento en enseñar estas actividades gracias a las ayudas ${ }^{1}$ y también nuestras investigaciones y las obras publicadas hacen hincapié en ello. Las ayudas de los profesores o de los propios compañeros son un punto clave para que estos aprendizajes sean colectivos. Debemos transmitir la idea de que estas ayudas se aprenden progresivamente y que el éxito de los aprendizajes depende en gran medida de ellas.

\section{¿Durante su paso por Brasil, como percibió usted la relación de la Universidad en} lo referente a la Gimnasia? ¿Cómo se encuentra esta relación en su país, España?

He detectado una estructura sólida en torno a la gimnasia y la acrobacia. El Foro Internacional de Gimnasia General, organizado por el grupo de investigación de la FEF-

\footnotetext{
${ }^{1}$ Sobre este assunto sugerimos a leitura destas obras: GERLING, I.E. Teaching children's gymnastics. Maideanhead: Meyer \& Meyer, 2009; HOLVOET, P. Studies on safety and equipament development. IN: JEMNI, M. et al. The Science of Gymnastics. Routledge, Francis and Taylor, 2011. Conexões: revista da Faculdade de Educação Física da UNICAMP, Campinas, v. 10, n. 3, p. 235-239, set./dez. 2012 ISSN: 1983-9030
} 
UNICAMP $^{2}$, es buena prueba de ello. Además, he notado mucha preocupación acerca de los aprendizajes, de los dispositivos materiales y de su evolución. Así mismo debo destacar la gran participación en los diferentes cursos de profesores y alumnos de Brasil y de otros países de América latina.

La gran crisis que sufre España se resiente también en la Universidad. La necesidad de que el profesorado tenga que realizar cada curso más horas de docencia repercute directamente en el grado de especialización del profesorado. Y con el Plan Boloña que ha reducido la carrera (el Grado) a 4 años, todas las asignaturas de han visto recortadas en su número de créditos, lo que significa menos horas presenciales y menor aprendizaje de los alumnos. De cara a los deportes gimnásticos, es otra desventaja más. Está claro que la especialización se debe buscar y exigir a las Federaciones Deportivas y la gimnasia en la Universidad se debe enfocar al ámbito educativo ya actividades acrobáticas extraescolares.

\section{Como profesional del área, ¿cuáles cree usted que son los reales desafíos de la educación física en el siglo XXI?}

Mi opinión es que la Educación Física en España se ha teorizado mucho después de tantos años en los que se enseñaba tan solo práctica. Debemos reivindicar más horas de práctica y también que se cumplan los contenidos que recogen la ley. Y de cara a la inclusión de la gimnasia en la Educación Física, debemos insistir en la necesidad de que los profesores realicen cursos de formación en los que actualicen sus conocimientos y se vean capaces de incluir las habilidades gimnásticas en sus sesiones prácticas. Hay que reconocer también la escasa dotación de material gimnástico en los centros escolares; queremos decir un material mínimo para poder llevar a cabo una unidad didáctica de acrobacia o gimnasia.

Como sabemos, la Educación Física es absolutamente necesaria para crear hábitos saludables en nuestros alumnos.

\section{¿Qué autores recomendaría para lectura en tiempos de exponencial aumento de la literatura acerca de la educación física y la sociedad?}

\footnotetext{
${ }^{2}$ A sexta edição do Fórum Internacional de Ginástica Geral (FIGG) foi realizada em julho em CampinasSP.

Conexões: revista da Faculdade de Educação Física da UNICAMP, Campinas, v. 10, n. 3, p. 235-239, set./dez. 2012. ISSN: 1983-9030
} 
En España seguimos los resultados de las investigaciones de M. García Ferrando en lo que se refiere a Hábitos Deportivos de los españoles en las diferentes Comunidades Autónomas; son estudios longitudinales que tienen en cuenta también el profesorado, las instalaciones deportivas, las tendencias actuales, etc. Y nos orientan hacia el futuro de la Educación Física en nuestro país.

Y concretamente, me tengo que referir de nuevo a la gimnasia artística y ahí tengo que comentar que existen bastantes obras en francés referidas al ámbito educativo y al competitivo $^{3}$; también en inglés por autores norteamericanos, pero me temo, referidos más al ámbito competitivo. En español, los libros de Vernetta y López Bedoya de la Universidad de Granada abordan el Acrosport (consultar también PEREZ GALLARDO; AZEVEDO, 2007) ${ }^{4}$ y la gimnasia en el contexto escolar y en Portugal, Carlos Araújo (2003), con su Manual de Ayudas en nuestra misma línea de trabajo. También tenemos obras referentes al Trampolín, al Circo $^{5}$ y la Acrobacia que son interesantes puntos de partida para poder incluir estos contenidos en la escuela o en clubes.

\section{¿Desea usted hablarnos de algún tema que no ha sido abordado en esta entrevista?}

Sí, me gustaría recordar la necesidad de que los resultados de las diferentes investigaciones y tesis llevadas a cabo en la gimnasia y la acrobacia en la Universidad reviertan en el ámbito educativo, competitivo e incluso en la iniciación deportiva. Es decir, acercar la investigación en la Universidad a la práctica directa con nuestros alumnos y así intentaremos mejorar la práctica docente.

\section{CONSIDERAÇÕES DOS ENTREVISTADORES}

Enfim, como podemos observar nestas breves linhas, apesar da tradição e da ampla produção acadêmica sobre a ginástica, e sobre a Educação Física de um modo mais

\footnotetext{
${ }^{3}$ Entre elas os entrevistadores destacam o clássico: LEGUET, Jacques. Actions motrices en gymnastique sportive. Paris : Vigot, 1985; e também: BOURGEOIS, Michel. Gymnastique Sportive: perspectives pédagogiques - école-club. Paris : Vigot, 1980, p.135-140; ROBIN, J. F. ; HAUW, D. Actualité de la recherché en activités gymniques et acrobatiques. Paris EPS, Paris, n. 39, 1998.

${ }^{4} \mathrm{Ou}$ ainda a famosa obra: POZZO, Thierry, STUDENY, Christophe. Théorie et pratique des sports acrobatiques. Paris ; Vigot, 1987.

${ }^{5} \mathrm{Na}$ literatura brasileira podemos mencionar: BORTOLETO, M A. C. et al. Introdução à pedagogia das atividades circenses. Jundiaí: Fontoura, Jundiaí, 2008; DUPRAT, Rodrigo Mallet; PEREZ GALLARDO, J. S. Artes circenses no âmbito escolar. Unijuí: Ed. da Unijuí, 2010.; BORTOLETO, M. A. C. et al. Introdução à pedagogia das atividades circenses. Jundiaí: Fontoura, 2010. v. 2.

Conexões: revista da Faculdade de Educação Física da UNICAMP, Campinas, v. 10, n. 3, p. 235-239, set./dez. 2012.
} 
geral, ainda temos muito caminho por percorrer e muitos interrogantes e desafios por superar.

\section{REFERÊNCIAS}

ARAÚJO, C. Manual de ayudas en gimnasia. Barcelona: Paidotribo, 2003.

ESTAPÉ, E. T. La acrobacia en gimnasia artística: su técnica y su didáctica. Barcelona: INDE, 2002.

PEREZ GALlARDO, J. S.; AVEZEDO, L. H. R. Fundamentos básicos da ginástica acrobática competitiva. Campinas: Autores Associados, 2007. 